CIC. Cuadernos de Información y Comunicación ISSN: 1135-7791

http://dx.doi.org/10.5209/CIYC.60687

\title{
La retención de usuarios en los videojuegos con multijugador masivo: Una analogía entre las motivaciones sociales que influyen en el ámbito lúdico y educativo
}

\author{
Jorge Osorio; Nahum Álvarez; Federico Peinado
}

Recibido: 30 de mayo de 2018 / Aceptado: 10 de junio de 2018

Resumen. Los géneros de videojuegos basados en dinámicas multijugador están sostenidos por la dimensión social inherente a estas. Esta dimensión contribuye de manera evidente en su éxito o fracaso y por tanto los creadores la consideran atentamente durante el diseño previo de la experiencia.

Comparando estas comunidades con las que se dan en el ámbito educativo encontramos paralelismos en las estructuras sociales de ambos sistemas, lo que propicia la extrapolación y aplicación de los resultados de investigación sobre videojuegos a las aulas universitarias.

Esta adaptación se plantea a través de un plan integral de acciones para potenciar el rendimiento y el aprendizaje del alumnado. Estas acciones se derivan de los métodos desarrollados en investigaciones previas con el objetivo de potenciar la estabilidad y la integración de los usuarios en comunidades de videojuegos.

Palabras clave: videojuegos, entorno educativo, motivaciones de uso, juegos multijugador online.

[en] Retention of users in video games with massive multiplayer: an analogy among the social motivations that influence the public and educational scope

\begin{abstract}
Video game genres based on multiplayer dynamics are sustained by their inherent social dimension. This dimension clearly contributes to the game's success or failure, therefore creators consider it very carefully during the previous design of the experience.

Comparing these communities among those from the educative environment we find correspondences between the social structures of both systems, which favor extrapolation and application of research results on video games to University's lecture rooms.

This adaptation is outlined on a comprehensive action plan to strengthen students' performance and learning. These actions originate in the methods developed in previous research with the purpose of fostering stability and integration of the users in video game communities.

Keywords: videogames, instructional context, use motivations, multiplayer online games.
\end{abstract}

Sumario. 1. Introducción. 2. Marco teórico. 2.1. Comunidad virtual. 2.2. Liderazgo. 2.3. La Teoría de la Autodeterminación. 2.3.1. Motivación extrínseca. 2.3.2. Motivación intrínseca. 3. Metodología. 4. Resultados. 4.1. Planificación de contenido. 4.2. Videojuegos derivados de otros productos culturales: Contenido educativo relacionado con la realidad. 4.3. Sistemas de integración e implicación. 4.4. Sensación de libertad. 4.5. Inmersión. 4.6. Orientador. 5. Discusión. 6. Conclusión. 7. Agradecimientos. Referencias.

Cómo citar: Osorio, J.; Álvarez, N.; Peinado, F. (2018). La retención de usuarios en los videojuegos con multijugador masivo: Una analogía entre las motivaciones sociales que influyen en el ámbito lúdico y educativo, en CIC. Cuadernos de Información y Comunicación 23, 189-200. 


\section{Introducción}

El avance tecnológico de nuestra era y concretamente su orientación hacia la conectividad global a través de la comunicación interactiva ha proporcionado el cimiento para la aparición de ciberespacios donde las comunidades sociales invierten cada vez más su tiempo. Esto ha transformado la vida cotidiana pues en dichos ciberespacios surge la socialización y son convertidos por tanto en una alternativa a los espacios públicos tradicionales.

Dentro de estos entornos virtuales con mayor presencia percibida encontramos el caso de los videojuegos tridimensionales. En concreto el género "Massively Multiplayer Online Role-playing Game" (a partir de ahora MMORPG) ha sido el contexto donde se ha desarrollado la investigación previa a este artículo.

Los primeros indicios de conexión entre el contexto educativo y los videojuegos se justifican con los estudios realizados por Ryan, Deci et al. (2000 y anteriores) en diferentes contextos donde se muestran paralelismos conductuales entre campos como el ámbito educativo, el laboral, el religioso etc.

En cuanto a la metodología, la investigación se basó en un formato de análisis triple sobre las conductas, las motivaciones asociadas a ellas y los factores de influencia que tuvieron una afectación directa o indirecta tratando de identificar, catalogar y relacionar cada elemento con la estructura conductual Una vez realizado este primer paso se plantearon modos de promover comportamientos a través de un conjunto de métodos aplicables a las comunidades buscando provocar comportamientos que derivaran en captación y retención de usuarios.

\section{Marco teórico}

\subsection{Comunidad virtual}

La observación directa que efectuó Rheingold del comportamiento en línea en todo el mundo durante una década le ha llevado a la conclusión de que la tecnología CMC (Comunicaciones mediadas por computadora), cuando se hace accesible públicamente, es utilizada para construir comunidades virtuales.

Una de las posibles explicaciones de este fenómeno, apunta el autor, es la necesidad de integrarse en comunidad que crece en el interior del ser humano en la medida en que cada vez más desaparecen espacios públicos informales en el mundo real (Rheingold, 1993:21).

Esta integración es un punto importante ya que el ser humano es un ser social y por ello tiende a organizarse en grupos a través de la socialización. Sin duda esta característica es independiente del contexto en el que se analice y nos sirve como catalizador de los métodos para ser aplicados a distintos ámbitos.

\subsection{Liderazgo}

Katz y Lazarsfeld en su obra "La influencia personal" parten de la idea de que lo importante es averiguar cómo las personas elaboran sus pensamientos y por qué estos pensamientos pueden cambiar. Se preguntan si los medios de masas son o no el mayor determinante de la decisión individual (Katz y Lazarsfeld, 1955:27). 
Se analizan por tanto las motivaciones y razones que llevan a los individuos a compartir sus opiniones con aquellos que los rodean a través de cuatro aspectos de análisis:

1. En primer lugar se considera como valor instrumental aquello que se genera compartiendo las opiniones y actitudes con quien una persona desea identificarse. $Y$ es que sucede que en la medida en que un grupo es atractivo para una persona, y en la medida en que ésta desea ser aceptada como miembro de aquél, se siente motivada a aceptar los puntos de vista del grupo. Este primer aspecto nos resulta muy útil como base y criterio de orientación pues si conseguimos generar ese atractivo y ese sentimiento de grupo en un aula, teóricamente los sujetos se verán predispuestos a obtener conocimientos y aumentará el rendimiento de los mismos.

2. En segundo lugar encontramos que el grupo proporciona una realidad social al individuo pues lo que existe como realidad para el sujeto se encuentra determinado en alto grado por lo que socialmente es aceptado como realidad sin perjuicio de basarse en los hechos físicos. Por tanto la realidad no es absoluta sino que varía en función de a qué grupo se pertenezca motivando a los sujetos a adquirir el enfoque de realidad aceptada por el grupo. (Katz y Lazarsfeld, 1955:58). De nuevo este segundo aspecto nos muestra la utilidad que posee el sentimiento grupal para el aprendizaje. Por tanto trataremos de que los conocimientos impartidos sean no sólo comprendidos si no con que conlleven una aplicación a la realidad para que de ese modo el alumnado como grupo comparta dichos conocimientos y los asuma de forma horizontal.

3. En tercer lugar se evalúa la interacción. En el trabajo realizado por Sherif (Sherif, 1952), se extrae la idea de que las personas interactúan con cada uno de los demás respecto a un problema particular que se refiere a todos, desarrollando una solución colectiva a dicho problema para crear una opinión, actitud, decisión o acción que después es acogida en común. Por tanto el alumnado no solo debe implicarse y rodearse del conocimiento impartido, también tienen que existir problemáticas relacionadas con el mismo que les afecten colectivamente y se deben ofrecer vías de acercamiento colaborativas para potenciar el sentimiento de comunidad entre ellos.

4. En cuarto lugar otra explicación para entender por qué las opiniones de las personas coinciden con las de aquellas que las rodean es la atracción de los valores compartidos. Robert K. Merton denomina a este fenómeno como valor homófilo (Merton, 1951).

A partir de estos cuatro aspectos se formulan sus ideas en torno a la figura del líder de opinión. En sus investigaciones revelan que al preguntarle a la gente por qué cambia su opinión sobre distintos aspectos de su vida en general, la gente contesta que el cambio fue motivado por la influencia de otras personas. Resulta pues la fuente predominante para el cambio de opinión.

\subsection{La Teoría de la Autodeterminación}

El modelo seleccionado para crear el protocolo de análisis aplicado en la investigación es la Teoría de la autodeterminación de Ryan \& Deci (2000) (TAD de aquí en adelante). La investigación relacionada con esta teoría se centra en el estudio del 
contexto social y las condiciones que se imponen al individuo. Estas condiciones tienden a potenciar y facilitar o por el contrario prevenir y entorpecer, los procesos que llevan a la auto-motivación o más conocidamente a las motivaciones intrínsecas.

Entienden por necesidad básica (sea fisiológica o psicológica) "un estado energizante que, si es satisfecho, conduce hacia la salud y el bienestar, pero si no es satisfecha, contribuye a la patología y al malestar. Por tanto, hemos propuesto que las tres necesidades básicas de competencia, autonomía, y de relacionarse tienen que ser satisfechas durante todo el decurso de la vida para que un individuo experimente un sentido continuo de integridad y bienestar." (R\&D 2000:10)

En su investigación señalan tres tipos de necesidades psicológicas innatas en el ser humano: competencia, autonomía y relación. Estas necesidades son el inicio de la estructura conductual que desencadena la acción.

La necesidad de autonomía se interpreta como un tipo de necesidad que causa sentimiento de voluntariedad en el sujeto motivado. Está relacionada con el libre albedrío y se centra en las percepciones del sujeto que tienden a generarle la necesidad de realizar una acción con diferentes expectativas de satisfacción interna como la obtención de diversión o la curiosidad.

La necesidad de competencia se centra en la potenciación de la autoestima en base a la demostración de las aptitudes del sujeto respecto a una acción. Puede tratarse de una demostración de cara a su comunidad o una demostración para sí mismo.

La necesidad de relación es un tipo de necesidad que se centra en la satisfacción y la seguridad que le otorga al sujeto la acción de socializar.

En la parte intermedia del proceso aparecen los factores de influencia relacionados con la necesidad los cuales afectan al desarrollo de la conducta y pueden convertirse en motivaciones que refuerzan, frenan y en definitiva orientan de un modo determinado la aparición de la acción.

\subsubsection{Motivación extrínseca}

La motivación extrínseca tiene como característica esencial la obtención de resultados que son separables de la actividad motivada para obtenerlos, a diferencia de la intrínseca que busca la satisfacción en la propia actividad o conducta motivada por el placer o valor que aporta al sujeto el simple hecho de realizarla. De acuerdo con la TAD, la cuestión real es cómo los individuos adquieren estas motivaciones y cómo éstas afectan a la persistencia, la conducta y el bienestar. En base a estos elementos se pueden observar diferentes estadios de motivación extrínseca en función de cuánto se ha internalizado la conducta motivada extrínsecamente.

Como criterios de clasificación utilizan el grado de autodeterminación de cada conducta motivada, el estilo regulatorio específico, el locus de causalidad percibido y los procesos regulatorios asociados al estilo.

\subsubsection{Motivación intrínseca}

En el estadio más internalizado se sitúan las motivaciones de carácter intrínseco. Son aquellas que reflejan las tendencias del sujeto a realizar conductas motivadas por el simple placer de realizarlas. Surgen del propio sujeto y se relacionan con la búsqueda de novedad, el desafío, la ejercitación de las capacidades propias o la exploración y el aprendizaje entre otras. 
A través de un estudio realizado sobre estudiantes en edad escolar Ryan \& Connell (1989) identificaron que las motivaciones reguladas externamente hacían que los sujetos mostraran menos interés, valor y esfuerzo además de negar su responsabilidad sobre los resultados negativos culpando a los agentes motivadores (maestros en este caso). En varios estudios (Deci, Koestner \& Ryan, 1999) se demostró que toda expectativa de recompensa tangible reduce la motivación intrínseca. Además de ello factores como la amenaza, la fecha de cumplimiento o las metas impuestas, también reducen dicha motivación.

Para argumentar el enfoque contrario se muestran tres fragmentos extraídos de la obra de los autores:

"Empleando entrevistas a los padres, Grolnick y Ryan (1989) hallaron una mayor internalización de los valores relacionados con la escuela entre los niños cuyos padres apoyaban más el sentimiento de autonomía y de relacionarse. Strahan (1995) halló que los padres que apoyaban más la autonomía promovían una mayor identificación religiosa, como opuesta a la introyección, en sus hijos. Williams y Deci (1996), usando un diseño longitudinal, demostraron una mayor internalización de valores y prácticas bio-psico-sociales entre estudiantes de medicina cuyos instructores apoyaban más la autonomía." (R\&D, 2000:9)

"Ryan, Stiller \& Lynch (1994) mostraron que los niños que habian internalizado más completamente la regulación para mostrar conductas positivas relacionadas con la escuela eran aquellos que se sentian firmemente conectados con sus padres y maestros y cuidados por estos." (R\&D, 2000:9)

"Deci, Eghrari, Patrick, y Leone (1994) demostraron en un experimento de laboratorio que proporcionar una racional significativa de una conducta no-interesante, junto con el apoyo a la autonomía y al relacionarse, promovió su internalización e integración. Los contextos controladores producen menos internalización general, y la internalización que ocurre en esos contextos tiende a ser solo introyectada.

Estos fragmentos muestran lo que tratamos de perseguir: Una buena integración social para obtener una base sobre la que mejorar el rendimiento académico promoviendo la autonomía ya que el grado de internalización está directamente relacionado con el nivel de autonomía percibida por el sujeto y a su vez genera por parte de éste un mayor compromiso con la acción, una efectividad comportamental superior, una mayor persistencia volitiva, un incremento del bienestar subjetivo del sujeto y una mejor asimilación del individuo dentro de su grupo social.

Posteriormente estos hallazgos se extendieron a través de demostraciones en las que se obtuvieron resultados positivos en educación, salud, religión, ejercicio físico, activismo ambiental, relaciones íntimas, etc. Estos descubrimientos indican que la autonomía y la relación como sentimientos promovidos y potenciados afectan de forma positiva en la educación y otros campos por lo que se infiere la posibilidad de extrapolar de manera exitosa los métodos generados a través de la TAD en el campo de los videojuegos.

\section{Metodología}

Dado que la orientación para recabar datos se centra en la exploración y obtención de la mayor cantidad de información posible sobre el objeto de estudio, se opta por 
utilizar la entrevista en profundidad como método principal para el desarrollo del estudio. La estructura de análisis generada para ello tuvo como objetivo el registro, exposición y comprensión de los procesos conductuales motivados, los factores de influencia y los estilos regulatorios.

Para esta tarea se generó un protocolo de análisis basado en la TAD que fue aplicado sobre los discursos ofrecidos por los sujetos investigados. Dicho protocolo de análisis motivacional se compuso de cinco fases: Localización de párrafos, Necesidad predominante, Factores de influencia, Estilo regulatorio y Métodos derivados.

\section{Resultados}

La aplicación de resultados engloba los métodos que se derivan del protocolo de análisis motivacional. Se trata de una descripción más precisa sobre sus características y los factores de influencia con los que se relacionan así como un desarrollo corto sobre su aplicación en el ámbito educativo.

\subsection{Planificación de contenido}

Se trata de un método compuesto de varias estrategias para fomentar la retención y recaptación de jugadores. La extrapolación de este método por tanto buscará la integración e implicación del alumnado en el aula y el mantenimiento de la atención fomentado por el atractivo del contenido.

La planificación de contenidos es un método principalmente relacionado con la necesidad de autonomía documentándose en 10 de las 41 conductas asociadas a esta necesidad las cuales fueron registradas a través del protocolo. Suponen un $24,3 \%$ del total de casos analizados siendo un método aplicable en casi la cuarta parte del total de conductas motivadas registradas. Concretamente este método se asocia a un subtipo de necesidad de autonomía; la necesidad satisfacer la curiosidad.

El factor de influencia predominante es el propio contenido. Hemos documentado un total de 14 ocasiones en las que aparece suponiendo un 25,5\% de las 55 localizaciones de factores registradas. De manera secundaria se registra el tedio como factor asociado a este método registrándose en una única ocasión suponiendo un $1,8 \%$ del total.

El primer planteamiento se centra en la dosificación del contenido consistente en realizar previsiones sobre el consumo buscando equilibrio entre la saturación por exceso de contenido y el tedio generado por un ritmo lento en el desarrollo del mismo.

Como segundo planteamiento sería conveniente hacer seguimiento de las tendencias que más novedad o atractivo generen en los sujetos para observar su evolución y variación. Con ello recabaríamos datos sobre qué aspectos potenciar en la planificación de contenido y cuáles modificar o eliminar por sus efectos negativos.

Desde los datos obtenidos partiría la implementación de acciones: Charlas de expertos en el campo de conocimiento, excursiones culturales o juegos colaborativos de corta duración. Con ello se evitarían las rutinas diarias excesivamente extendidas en el tiempo y el flujo de contenido se equilibraría de tal manera que la percepción de novedad no desaparecería o no tendería a reducirse. 


\subsection{Videojuegos derivados de otros productos culturales: Contenido educativo relacionado con la realidad}

Este método consiste en la creación de videojuegos en base a componentes relacionados con la cultura de masas. Se registra entre las conductas asociadas a la necesidad de autonomía en un único caso de 41 suponiendo un 2,43\% del total. De nuevo el subtipo de necesidad es la curiosidad por el contenido.

La experiencia personal es el factor de influencia predominante documentándose en 7 ocasiones y suponiendo un $12,7 \%$ del total de 55 localizaciones. Consiste en las vivencias y contactos anteriores que el sujeto tiene con un tema determinado los cuales pueden propiciar su predisposición a prestar atención si el producto cultural tiene relación con su experiencia.

Aplicado al campo de la docencia se propone como método ofrecer un fuerte contraste entre el contenido teórico y la realidad actual a modo de gancho de atención para el alumnado. Consistiría en recabar información sobre los temas de interés que sean tendencia llevar el conocimiento que se pretende impartir hasta dichos temas. Con ello no sólo se conseguiría captar la atención si no que se mostraría la utilidad del contenido impartido.

Por ejemplo, si se realiza una estructura relacional, podríamos ofrecer al alumnado la utilidad de las matemáticas asociándolas a la programación y ésta asociándola a la creación de programas y aplicaciones como YouTube o Minecraft.

\subsection{Sistemas de integración e implicación}

Este método consiste en la aplicación de un conjunto de diseños orientados a potenciar el rendimiento a través de la integración en el grupo y la estabilidad del mismo. En la investigación dicho conjunto se ha asociado a conductas derivadas de necesidades del tipo relación. Concretamente se han localizado dos casos entre 30 suponiendo un $6,66 \%$ sobre el total de analizados basados en una necesidad de relación.

El factor de influencia predominante en ambos casos es el círculo social al que pertenece el sujeto localizándose en 26 ocasiones lo que supone el 56,52 \% del total de 46 registros de factores de influencia asociados a conductas cuya necesidad predominante es de relación.

La aplicación consiste en la generación de un ranking entre aulas de diferentes centros cuya valoración sea medida por el progreso global consistente en la suma del progreso de cada miembro. De ese modo se potencia la auto-percepción del usuario como miembro valioso además del sentimiento de valía en conjunto respecto a otras comunidades. La medición se realizaría sobre una problemática colectiva similar planteada en cada aula.

Este método fomentaría la externalización de la competitividad reduciendo el nivel de rivalidad dentro del aula y reforzando el sentido de pertenencia a ella. El alumno se integraría a través del objetivo colectivo y obtendría mejor rendimiento en base a la presión social del entorno y a la necesidad de aunar esfuerzos para resolver la problemática colectiva. Dicha resolución debe ser alcanzada únicamente si cada sujeto maneja el conocimiento que se pretende que aprenda y todos participan en el camino hacia la solución. 


\subsection{Sensación de libertad}

Conocida técnicamente como ilusión de agencia, la sensación de libertad es una característica que figura como factor de atracción y retención en los análisis realizados. Se localiza en tres ocasiones, dos de ellas en relación con necesidades de autonomía y una tercera que deriva de una necesidad de competencia. Por tanto dentro de las conductas basadas en necesidades de tipo autonomía representa un 4,87\% sobre el total de 41 y en el caso relacionado con la competencia representa un $7,14 \%$ sobre el total de 14 conductas asociadas a necesidades de competencia.

El factor de influencia predominante en el caso relacionado con la necesidad de competencia es el diseño del videojuego. Se localiza en 3 ocasiones de un total de 16 suponiendo un $18,75 \%$. Cada caso relacionado con la necesidad de autonomía ofrece un factor de influencia diferente. Aparece el cierre del servidor de juego que se documenta en 2 ocasiones de 55 representando un 3,63\% del total y la comunidad en el segundo caso localizándose en 16 de 55 ocasiones (29\% de los factores de influencia documentados).

La acción que se propone es que las actividades dentro de las aulas deberían basarse en la ilusión de libertad ofreciendo cierto control de los grupos sobre el contenido a tratar, permitiéndoles elegir un tema de desarrollo relacionado, la posibilidad de realizar una investigación corta sobre la materia o la creación de una batería de debates a lo largo del curso que les hagan razonar las ideas y conocimientos de la asignatura. No se considera libertad auténtica porque al margen de las opción de avance que seleccione el grupo el orientador debe limitar las opciones de forma sutil redirigiendo las propuestas que no sean viables.

\subsection{Inmersión}

Este mecanismo de evasión es frecuentemente utilizado por los jugadores y tiene una relación proporcional entre la inmersión percibida por el sujeto y la evasión que obtiene jugando. Dentro de los videojuegos se potencia esta percepción promoviendo actividades emergentes de manera indirecta consistiendo en el diseño de estructuras jugables que den pie a experiencias y narrativas no prediseñadas.

La extrapolación al ámbito educativo consistiría en ofrecer contenido no convencional durante el curso, cediendo en ocasiones la clase a expertos sobre la materia, planificando mesas redondas o visualizando documentales relacionados Con ello buscaríamos la implicación de los sujetos con la materia cursada tratando de cambiar su actitud de oyente a participante o lo que es lo mismo de público pasivo a público activo.

\subsection{Orientador}

La figura del orientador se documenta en los tres tipos de necesidad siendo un método muy recurrente y adaptable a diversas situaciones. Dentro de las conductas basadas en una necesidad de autonomía se registra en 10 de 41 conductas lo que representa un $24,39 \%$ del total. En cuanto a los factores de influencia, dentro de estos diez casos se localizan 2 factores de influencia distribuidos de forma equivalente: 5 registros de toxicidad y 5 registros de comunidad como factores de influencia lo que representa un $9,09 \%$ en cada factor sobre un total de 55 localizaciones. 
En el caso de la necesidad de competencia se registra en 8 conductas de 14 localizadas representando una presencia del $57,14 \%$ en las conductas de este tipo. El factor de influencia principal es la comunidad que se registra en 7 de 16 casos suponiendo un $43,75 \%$ de los factores de influencia registrados que se asocian a conductas basadas en necesidades de tipo competencia. Por otro lado la equipación del avatar aparece en un único caso suponiendo un $6,25 \%$ del total.

Por último en las conductas de tipo relación se registra en 10 de los 30 casos documentados suponiendo un $33,33 \%$. Respecto a los factores de influencia asociados a las conductas donde figura el método del orientador como instrumento de optimización aparecen 3 factores de influencia. El peso recae sobre la comunidad como factor de influencia al registrar en 8 ocasiones suponiendo un 17,39\% del total de 46 registros de factores de influencia. Los otros dos casos registran la toxicidad y el contenido del videojuego como factores de influencia suponiendo un $2,17 \%$ cada uno sobre el total.

En conjunto se puede observar que el factor de influencia predominante en el que basarse para aplicar este método es la comunidad. En la aplicación al campo educativo se han seleccionado las funciones del orientador que mejor se adaptan para optimizar el rendimiento dentro de las aulas y potenciar la atención del alumnado:

Gestor: La figura del orientador debe encargarse de que cada alumno se integre en el grupo observando el entorno y previniendo la marginación de cada sujeto a través de la reorientación conductual y el asesoramiento personal. Debe por tanto identificar los perfiles de alumnos lo antes posible para prevenir este tipo de comportamientos.

Instructor: La labor de instrucción es la parte esencial del profesorado. Cada orientador debe llevar sus esfuerzos a conseguir ser percibido más como un guía y un soporte que como una figura superior en términos de conocimientos pues tratamos de implicar al alumno con el contenido. Volvemos a la idea de horizontalidad en la comunicación frente a la estructura jerárquica convencional que distancia al alumno del profesor.

Mediador: La función de mediador busca prevenir y solucionar los conflictos antes de que su gravedad aumente. Debe tomar medidas disciplinarias si se da la necesidad, ya que normalmente el conflicto empieza entre dos individuos o un grupo reducido de sujetos frente a un único individuo. Si no se interviene la toxicidad se extiende y acaba obligando a escoger bando a toda la comunidad generando rencillas y divisiones además de provocar nuevos conflictos lo que desencadena incomodidad y malestar generalizado.

\section{Discusión}

Como en todo método experimental, debemos tratar de analizar sus componentes en profundidad para comprender cuáles pueden ser sus limitaciones. En primer lugar la aplicación de los métodos descritos se considera insuficiente si cualquiera de los dos criterios siguientes no se aplican o se aplican incorrectamente:

- Los métodos deben plantearse de tal manera que impliquen el contenido, una gamificación del aula que los integre de manera independiente tendrá una efectividad inferior o nula, como puede verse en Rowe et al. (2009). 
- Los métodos deben aplicarse en conjunto y con un orden concreto respondiendo como un plan integral:

En primer lugar se debe fomentar el sentimiento de grupo aplicando el método que potencia la ilusión de libertad dialogando sobre la temática de la clase y llegando a un consenso sobre la idea concreta en la que consistirá el trabajo colectivo posterior.

En segundo lugar se debe despertar el interés por la materia a través de los métodos de inmersión y el contenido educativo relacionado con la realidad En tercer lugar se plantearía el trabajo o problemática colectiva sobre la que trabajarían durante el curso en los horarios de la asignatura, para ello se aplicarían los sistemas de integración e implicación entre el alumno y el contenido educativo.

Como ejes transversales a cada paso se aplicaría la planificación del contenido y las funciones del orientador para tratar de reorientar y encauzar el curso cuando se detecten conductas indeseadas.

Las limitaciones del conjunto aplicable se concentran principalmente en las características del entorno:

En primer lugar se considera que su implementación solo sería viable en grupos reducidos de alumnos pues la integración como un solo grupo se diluye cuanto mayor es el número de sujetos que lo forman. Además las funciones del orientador en un aula numerosa es completamente inefectiva o como mínimo insuficiente, dado que ejercer gestión y vigilancia y mediación es solo posible en grupos reducidos de no más de diez alumnos.

En segundo lugar el estudiante debe tener un mínimo de interés por la materia, hecho que se presupone que ocurre de una manera mucho más general en el ámbito universitario, donde la obligatoriedad para estudiar desaparece y los sujetos entran voluntariamente a adquirir conocimientos.

\section{Conclusión}

La flexibilidad que ofrece la TAD, tal como se muestra en el marco teórico nos ha permitido extrapolar los resultados de un campo de aplicación a otro ya que tras ser aplicada a los MMORPG obteniendo unos resultados satisfactorios, éstos han sido fácilmente implementados al campo de la educación. Sin embargo no podemos ratificar de forma empírica esta hipótesis todavía, dado que no poseemos resultados de aplicación que demuestren estos paralelismos estructurales del comportamiento humano en ninguno de los dos ámbitos.

Aunque la TAD sí que ha mostrado su aplicabilidad en diferentes campos y concretamente ha sido usada para analizar las motivaciones de estudiantes de diferentes rangos de edad, estamos todavía en una etapa de exploración. Estos estudios son un indicio que nos lleva a predisponernos a favor de que la extrapolación entre videojuegos y educación pueda ser realmente efectiva.

El estudio motivacional puede ofrecer ventajas en cuanto a pruebas de métodos dentro de los videojuegos por ser un entorno cerrado, medible y sobre todo controlable a niveles muy superiores si se compara con otros contextos para realizar trabajos de campo. El videojuego es comparable a un laboratorio pero con la peculiaridad de 
que se trata de un laboratorio social. De hecho llevamos años viendo cómo cobra cada vez más importancia el uso de las métricas obtenidas para optimizar la experiencia de los usuarios que interactúan con videojuegos.

El primer obstáculo que debe salvarse es la demostración de su utilidad para testear métodos que puedan ser aplicados a la enseñanza. Lo que sugiere la realización de un proyecto de investigación cruzada orientada a analizar los paralelismos estructurales. Una vez obtenidos, los resultados deberían mostrar las mismas reacciones y comportamientos motivados ante los condicionamientos sufridos por los sujetos de uno y otro campo.

Es posible que estas reacciones no se den en la misma medida, pero parece plausible que aplicar un método que potencie la integración grupal en ambos ámbitos sí debería integrar a los sujetos en sus respectivas comunidades, siempre que la estructura de sus conductas y sus necesidades sean también similares.

\section{Agradecimientos}

Este trabajo se apoya parcialmente en el proyecto ComunicArte: Comunicación Efectiva a través de la Realidad Virtual y las Tecnologías Educativas, financiado por las Ayudas Fundación BBVA a Equipos de Investigación Científica 2017, y el proyecto NarraKit VR: Interfaces de Comunicación Narrativa para Aplicaciones de Realidad Virtual (PR41/17-21016), financiado por las Ayudas para la Financiación de Proyectos de Investigación Santander-UCM 2017.

\section{Referencias}

Deci, E.L., Eghrari, H., Patrick, B. C., \& Leone, D. R. (1994). Facilitating internalization: The self-determination theory perspective. Journal of Personality, 62, pp. 119-142.

Deci, E. L., Koestner, R., \& Ryan, R. M. (1999). A meta-analytic review of experiments examining the effects of extrinsic rewards on intrinsic motivation. Psychological Bulletin, 125 , pp. 627-668.

Grolnick, W, S., \& Ryan, R. M. (1989). Parent styles associated with children's self-regulation and competence in school. Journal of Educational Psychology, 81, pp. 143-154.

Katz, E. y Lazarsfeld, P. (1955). La Influencia Personal. Barcelona: Hispano Europea.

Merton, R. K. (1951). Patterns of Social Life: Explorations in the Sociology of Housing. New York: Columbia University Bureau of Applied Social Research.

Rheingold, H. (1996). La comunidad virtual: Una sociedad sin fronteras. Barcelona: Gedisa. Rowe, J. P., McQuiggan, S. W., Robison, J. L., \& Lester, J. C. (2009). "Off-task behavior in narrative-centered learning environments". S. Craig \& D. Dicheva (Eds.) Proceedings of the 14th International Conference on Artificial Intelligence in Education pp. 99-106. Berlin: Springer-Verlag.

Ryan, R. M., \& Deci, E. L. (2000). Deci Self-Determination Theory and the Facilitation of Intrinsic Motivation, Social Development, and Well-Being. American Psychologist Vol. 55, No. 1. pp. 68-78.

Ryan, R. M., Stiller, J., \& Lynch, J. H. (1994). Representations of relationships to teachers, parents, and friends as predictors of academic motivation and self-esteem. Journal of Early Adolescence, 14, pp. 226- 249. 
Sherif, M. (1952). Social factors in perception. New York: Eds. Readings in social psychology, Henry Holt.

Strahan, B. J. 1995. Marriage, family and religion. Sydney, Australia: Adventist Institute.

Williams, G. C., \& Deci, E. L. 1996. Internalization of biopsychosocial values by medical students. Journal of Personality and Social Psychology, 70, pp. 767-779. 